\title{
Fenofibrate decreases the bone quality by down regulating Runx2 in high-fat-diet induced Type 2 diabetes mellitus mouse model
}

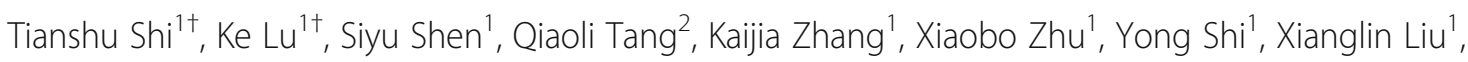
Huajian Teng ${ }^{1,3}$, Chaojun $\mathrm{Li}^{2^{*}}$, Bin Xue $2,4,5^{*}$ and Qing Jiang ${ }^{1,3^{*}}$

\begin{abstract}
Background: This study is to investigate the effect of fenofibrate on the bone quality of Type 2 diabetes mellitus (T2DM) mouse model.

Methods: T2DM mouse model was induced by high-fat-diet, and the mice were treated with fenofibrate (100 mg/ $\mathrm{kg}$ ) (DIO-FENO) or PBS (DIO-PBS) for 4 weeks. The bone microstructure and biomechanical properties of femora were analyzed by micro-CT and 3-Point bending test. The protein expression was detected by immunohistochemical staining and Western blot. The cell apoptosis was evaluated by TUNEL staining. The Bcl2, caspase 3, and osteoblast marker genes were detected by RT-qPCR.

Results: The biomechanical properties of bones from DIO-FENO group were significantly lower than those in the control and DIO-PBS groups. Besides, the trabecular number was lower than those of the other groups, though the cortical porosity was decreased compared with that of DIO-PBS group because of the increase of apoptotic cells. The expression of osteocalcin and collagen I were decreased after treatment with fenofibrate in T2DM mice. Moreover, the cell viability was decreased after treated with different concentrations of fenofibrate, and the expression of Runx2 decreased after treated with high dose of fenofibrate.
\end{abstract}

Conclusion: Fenofibrate decreases the bone quality of T2DM mice through decreasing the expression of collagen I and osteocalcin, which may be resulted from the down regulation of Runx2 expression.

Keywords: Fenofibrate, Bone quality, Runx2, Type 2 diabetes mellitus

\section{Background}

Type 2 diabetes mellitus (T2DM) is a global disease characterized by abnormal metabolism of blood glucose, lipid and protein caused by insulin resistance or abnormal insulin secretion $[1,2]$. In addition to increased blood glucose, patients with T2DM also have

\footnotetext{
*Correspondence: licj@nju.edu.cn; xuebin@nju.edu.cn; qingj@nju.edu.cn ${ }^{\dagger}$ Equal contributors

${ }^{2}$ State Key Laboratory of Pharmaceutical Biotechnology and Jiangsu Key Laboratory of Molecular Medicine and School of Medicine, Nanjing University, No. 22 Hankou Road, Gulou District, Nanjing, Jiangsu Province 210093, China

${ }^{1}$ Department of Sports Medicine and Adult Reconstructive Surgery, Drum Tower Hospital, School of Medicine, Nanjing University, No. 321 Zhongshan Road, Nanjing 210008, People's Republic of China

Full list of author information is available at the end of the article
}

complications, such as glomerular sclerosis, diabetic foot and brittle fractures [3]. Schwartz et al. found that patients with T2DM have a higher risk of hi p fracture [4]. Bonds et al. reported that bone mineral density (BMD) of T2DM patients increased by $5-10 \%$ compared to nondiabetic patients of the same age [5]. However, the pathogenesis of diabetes related bone disease is not clear, and some reports indicated that it may be associated with the increased secretion of advanced glycation end products [6-8], sclerostin [9] and adipokines [10, 11]. On the other hand, it may be related with other complications associated with T2DM, such as retinopathy, which increased the likelihood of falling [12]. 
The multiple complications of T2DM may affect each other and reduce the survival rate of patients. For example, in about $65 \%$ of T2DM patients, who die of cardiovascular disease, dyslipidemia is one of its important predisposing factors $[1,13]$. There are a large number of patients with T2DM associated with varying degrees of dyslipidemia characterized by triglyceride (TG) increase and high density lipoprotein decrease [13, 14]. Bijelic et al. reported that the LDL cholesterol and triglycerides were significant risk factors for osteoporosis [15]. Yamaguchi et al. also reported that plasma HDLCholesterol levels were significantly and positively correlated with the absolute values of BMD [16]. This result may be related to the development of an inflammatory microenvironment that affects the differentiation and function of osteoblasts caused by the decrease of HDLCholesterol.

Fibrates are a class of drugs that can stimulate lipoprotein lipase (LPL) activity by lipolysis of TG in lipoproteins [13], and fenofibrate is one of the most commonly prescribed fibrates. The Fenofibrate Intervention and Event Lowering in Diabetes (FIELD) study showed that fenofibrate can effectively reduce blood TG levels and reduce the risk of total cardiovascular events [17]. And the use of fenofebrate is associated with a decrease in the rate of diabetic amputation and a reduction in retinal lesions $[18,19]$. Lee et al. found that gout patients taking fenofibrate may increase the risk of kidney stones [20]. However, there is no report about whether fenofibreate will have an impact on the skeletal system in the treatment of diabetic hyperlipidemia.

In this study, we established a high-fat-diet (HFD) induced T2DM mouse model with dyslipidemia, and evaluated the effects of fenofibrate on bone mass and abnormality in bone microstructure and function. The potential mechanism underlying the effects of fenofibrate was also investigated.

\section{Methods}

\section{Animals}

Two-week-old diet-induced obese (DIO)-C57/BL6 mice were provided by Nanjing Biomedical Research Institute of Nanjing University (NBRI, Nanjing, China). They were kept at room temperature $\left(20-25{ }^{\circ} \mathrm{C}\right)$ with a constant humidity $(55 \pm 5 \%)$ and free access to food and water in a $12 / 12 \mathrm{~h}$ light/dark cycle. All animal experiments were conducted in accordance with the Institutional Animal Ethics Committee and Animal Care Guidelines for the Care and Use of Laboratory Animals of Nanjing University.

\section{Establishment of T2DM model}

Feeding C57BL/6 J mice with HFD is a wellcharacterized model that results in hyperglycaemia, hyperinsulinemia, insulin resistance, defective islet compensation, and impaired glucose tolerance. For HFD groups $(n=12)$, mice were fed with a HFD (D12492, research diets, New Brunswick, NJ) containing 58.0\% fat, $16.4 \%$ protein, $25.6 \%$ carbohydrates for 10 weeks.

\section{Animal grouping and treatment}

After 10 weeks of feeding with HFD, some mice $(n=6)$ in the HFD groups were gavaged with fenofibrate (100 mg/kg, DIO-FENO) (Sigma, Germany) for 4 weeks [21], and other mice ( $n=6$ were treated with PBS (DIOPBS). However, for the control group $(n=6)$, routine diet (RD) was provided. At the end of the feeding period, mice were anesthetized with halothane. Tissues were harvested for analysis as described below.

\section{Microcomputed tomography (micro-CT) analysis}

The left femora dissected from the three groups were fixed with $4 \%$ paraformaldehyde for $24 \mathrm{~h}$, washed by PBS, and then scanned by a micro-CT scanner (SkyScan, Aarselaar, Belgium). X-ray voltage and current were set at $80 \mathrm{kV}$ and $80 \mu \mathrm{A}$, respectively, with a resolution of $18 \mu \mathrm{m}$ per pixel. Cross-sectional images of femur were used for 3dimensional histomorphometric analysis of trabecular bone. For the distal femur, the region of interest (ROI) selected for analysis was 5\% of femoral length from $0.05 \mathrm{~mm}$ below the growth plate to determine trabecular bone mineral density (Tb.BMD), trabecular bone volume per tissue volume (BV/TV), trabecular number (Tb. N), trabecular separation (Tb. Sp), and trabecular thickness (Tb. Th). Crosssectional images of the mid-diaphysis of femur were used for 3-dimensional histomorphometric analysis of cortical bones. For cortical bones, the ROI selected for analysis was $10 \%$ of femoral length in the mid-diaphysis of the femur to determine cortical bone mineral density (Ct.BMD), cortical thickness (Ct. Th), and cortical porosity (Ct. Po).

\section{Measurement of bone biomechanics}

The biomechanical properties of the bones were determined according to the procedures described by Mattila et al. [22]. The bones were subjected to three-point bending test on a universal testing machine (Instron Corp., CAT NO 2752-005, Canton, MA) until failure. Three-point bending strength was measured with a constant span length of $10 \mathrm{~mm}$. The compressive loading speed in all tests was $0.1 \mathrm{~mm} / \mathrm{s}$. The obtained load-time curve was converted into a load-displacement curve. Breaking force was defined as bending load at failure. Stiffness was calculated as the slope of the linear (elastic) part of the loaddisplacement curve. 


\section{Histological analysis}

The right femora were decalcified in 5\% EDTA in PBS for 2 weeks, embedded in paraffin after dehydration and coronally cut into $5 \mu \mathrm{m}$ sections. Then immunohistochemical staining of collagen I and TUNEL staining were performed as described below.

\section{Immunohistochemical staining}

In brief, bone sections were deparaffinized in xylene and rehydrated in diminishing concentrations of ethanol, followed by subsequent incubation in $3 \% \mathrm{H}_{2} \mathrm{O}_{2}$ for $10 \mathrm{~min}$ at room temperature to eliminate endogenous peroxidase activity. Antigen-retrieval was performed by heating the sections for 9 min in EDTA buffer ( $\mathrm{pH}$ 6.0). Thereafter, the slides were blocked with goat serum albumin (BOSTER, Pleasanton, USA) for $15 \mathrm{~min}$ at $37{ }^{\circ} \mathrm{C}$, and then incubated overnight at $4{ }^{\circ} \mathrm{C}$ with primary antibody specific for Collagen I (Abcam, Cambridge, USA). After rinsing, the slides were incubated for $1 \mathrm{~h}$ at $37{ }^{\circ} \mathrm{C}$ with secondary antibody of goat anti-rabbit IgG (HRPlabeled, Santa Cruz Biotech). After rinsing again, the sections were stained with DAB. Finally, the sections were rinsed in distilled water, and counterstained with haematoxylin. Finally, the area fraction of collagen I were developed and quantified by Image J version 1.6 (National institutes of Health, USA).

\section{TUNEL staining}

TUNEL assay was performed with an in situ cell death detection kit (G3250, Promega, USA) according to the manufacturer's protocol. Briefly, paraffin-embedded sections were deparaffinized, washed with PBS and pretreated with proteinase $\mathrm{K}(20 \mu \mathrm{g} / \mathrm{ml})$ for $15 \mathrm{~min}$. The equilibration buffer was applied directly to each section and incubated for $10 \mathrm{~s}$. Sections were then incubated with rTdT enzyme in a humidified chamber at $37{ }^{\circ} \mathrm{C}$ for $1 \mathrm{~h}$. After being labeled, sections were washed and mounted under coverslips. DNA fragmentation was detected by selecting four fields at $100 \times$ magnification in each section. All images were analyzed by Adobe Photoshop CS2 to quantify the signals. Data were expressed as the number of apoptotic cells per high-power field.

\section{Western blot}

Bone proteins were extracted from the samples after mechanical testing. After washing medullary cavity, the bones were ground into powder in liquid nitrogen, homogenized directly in RIPA buffer supplemented with $\mathrm{Na}_{3} \mathrm{VO}_{4}, \mathrm{NaF}, \mathrm{PMSF}$ and Cocktail, and then centrifuged at $4{ }^{\circ} \mathrm{C}$ for $10 \mathrm{~min}$ at $12,000 \times \mathrm{g}$. The resulting supernatant was separated by SDS-PAGE and transferred to membranes. After blocking, primary antibodies of antiosteocalcin (Santa Cruz Biotechnology, USA) anticollagen I (Abcam, Cambridge, USA) and anti- $\beta$-actin
(Santa Cruz Biotechnology, USA) were added and incubated overnight at $4{ }^{\circ} \mathrm{C}$. Then, the membranes were incubated with secondary antibody of goat anti-rabbit and anti-mouse IgG (HRP-labeled, Santa Cruz Biotech) in PBST for 40 min. Finally, specific bands were developed and quantified by Image J version 1.6 (National institutes of Health, USA.

\section{Cell line and cell culture}

Murine MC3T3-E1 pre-osteoblast cell line was were grown in $\alpha$-minimum essential medium ( $\alpha$-MEM) (Gibco BRL, Grand Island, NY, USA) with $10 \%$ fetal bovine serum and $1 \%$ penicillin-streptomycin (Gibco BRL) under $5 \% \mathrm{CO}_{2}$ atmosphere at $37{ }^{\circ} \mathrm{C}$.

\section{Cytotoxicity test}

To analyze the cytotoxicity of fenofibrate on MC3T3 cells, a CCK- 8 assay (Chengjian, NanJing, China) was used according to the manufacturer's instructions. Briefly, MC3T3 cells were plated in 96-well plates at a density of $5 \times 10^{3} /$ well. And then, solutions containing various concentrations of fenofibrate $(0,25,100,200 \mathrm{ng} / \mu \mathrm{L})$ were added. The standard protocol for assessing cell viability was then carried out.

\section{Quantitative PCR}

MC3T3 cells were plated in 12-well plates at a density of $1.8 \times 10^{5} / \mathrm{ml}$. After incubation for $12 \mathrm{~h}$, total RNA was extracted using TRIzol (Takara, Shiga, Japan). The cDNA was then synthesized from total RNA by a reverse transcriptase cDNA synthesis kit (Takara, Shiga, Japan). Quantitative PCR was carried out on a 7500 Real-Time PCR System (Applied Biosystems, Waltham, MA, USA) using SYBR Premix Ex Taq (Takara, Shiga, Japan). Forward and reverse primers were listed in Table 1. All data were normalized to $\beta$-actin and assays were performed in triplicate.

\section{Statistical analysis}

The data were expressed as mean \pm standard deviation (SD). Student's $t$-test and one-way ANOVA were used to assess the significance of differences using GraphPad Prism version 4.0. The post hoc analysis was also carried out. A $P$-value less than 0.05 was considered statistically significant.

\section{Results}

Fenofibrate causes a further decrease in bone mass of the HFD-induced T2DM mice

To determine the changes in bone biomechanics after fenofibrate treatment in T2DM mice, the three-point stress bending test and micro-CT analysis were performed, respectively. As shown in Fig. 1a and b, the femoral stiffness and the max load of the DIO-PBS group 
Table 1 Primer sequences used for quantitative PCR

\begin{tabular}{lll}
\hline Primer & Forward $\left(5^{\prime}-3^{\prime}\right)$ & Reverse $\left(5^{\prime}-3^{\prime}\right)$ \\
\hline Runx2 & AACGATCTGAGATTTGGGGC & CCTGCGTGGGATTTCTTGGTT \\
Osteocalcin & CTGACCTCACAGATCCCAAGC & TGGTCTGATAGCTCGTCACAAG \\
Spp1 & AGCAAGAAACTCTTCCAAGCAA & GTGAGATTCGTCAGATTCATCCG \\
Sp7 & ATGGCGTCCTCTCTGCTTG & TGAAAGGTCAGCGTATGGCTT \\
Bcl2 & GTCGCTACCGTCGTGACTTC & CAGACATGCACCTACCCAGC \\
Caspase3 & ATGGAGAACAACAAAACCTCAGT & TTGCTCCCATGTATGGTCTTAC \\
$\beta$-actin & CATGTACGTTGCTATCCAGGC & CTCCTTAATGTCACGCACGAT \\
\hline
\end{tabular}

decreased significantly compared with the RD group (F (2, 12) $=9.061, P=0.004$ for Fig. $1 \mathrm{a}$; and $F(2,13)=10.22$, $P=0.002$ for Fig. $1 b)$. The max load value of the DIOFENO group was significantly decrease compared to the DIO-PBS group $(P<0.05)$, while there was no significant difference in the bone stiffness values between these two groups.

In order to clarify the causes of reduced bone biomechanical properties after treatment with fenofibrate, micro-CT analysis was performed. As shown in Fig. 1c, the trabecular bone mass in DIO-FENO group was lower than that in the DIO-PBS group. There were no significant differences in trabecular bone related parameters such as $\mathrm{BMD}, \mathrm{BV} / \mathrm{TV}$, trabecular bone numbers, trabecular bone thickness, and trabecular bone separation between the DIO-PBS group and RD group. (Fig. 1d-h). This indicates that the bone quality decreased in the HFD-induced T2DM (DIO-PBS) mice, which means that the biomechanical properties of T2DM bones significantly decreased without bone mass change. It suggests that HFD-induced T2DM causes a change in bone hardness, leading to bone brittleness increase. Compared with RD group or DIO-PBS group, the trabecular number (Fig. 1f) (F $(2,13)=5.113$, $P=0.023)$ in the DIO-FENO group significantly decreased whereas the trabecular separation (Fig. 1h) (F $(2,14)=11.71, P=0.001)$ in the DIO-FENO group significantly increased $(P<0.05)$.

These results indicate that trabecular bone mass decreases in the T2DM mice after fenofibrate treatment, and thus leads to a decrease in bone biomechanics.

\section{Fenofibrate causes a decrease in bone porosity of the HFD-induced T2DM mice}

Bone fragility is significantly increased in diabetic patients, and the porosity of cortical bones has a

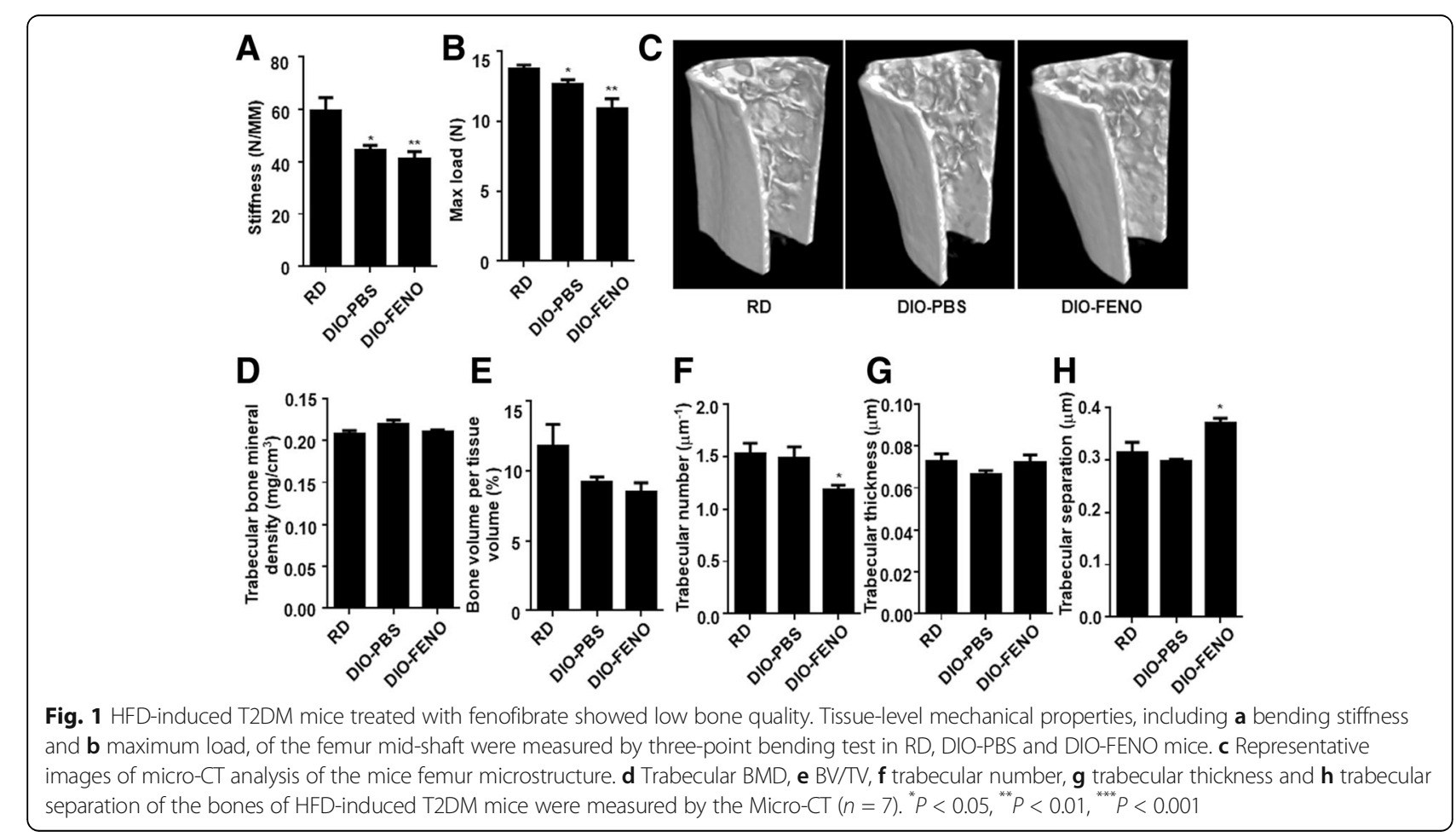


significant impact on brittle fractures [23]. To evaluate the microstructure of cortical bones from different groups, micro-CT was performed. There were no significant differences in cortical bone BMD and thickness (Fig. 2a and b) among the three groups. However, the cortical bone porosity in the DIO-PBS group was significantly increased $(P<0.05)$ (Fig. 2c) $(\mathrm{F}(2,10)=17.01$, $P=0.0006)$. After fenofibrate treatment, the cortical bone porosity in the DIO-PBS group was significantly decreased than the DIO-PBS group (Fig. 2c). No significant difference was found between the RD group and the DIO-PBS group. This indicates that the cortical porosity of HFD-induced T2DM mice was increased, which might cause the decrease of bone biomechanical properties. However, a contrary result showed that the cortical porosity was decreased after fenofibrate treatment compared with that of DIO-PBS group.

To further explore the mechanism of the contrary result in DIO-FENO group, TUNEL staining was performed. Cell apoptosis in the cortical bones of the DIO-FENO group significantly increased after treated with fenofibrate (Fig. $2 \mathrm{~d}$ and e). At the same time, the expression levels of $\mathrm{Bcl} 2$ and caspase 3 mRNA were significantly increased in the MC3T3 cells after treated
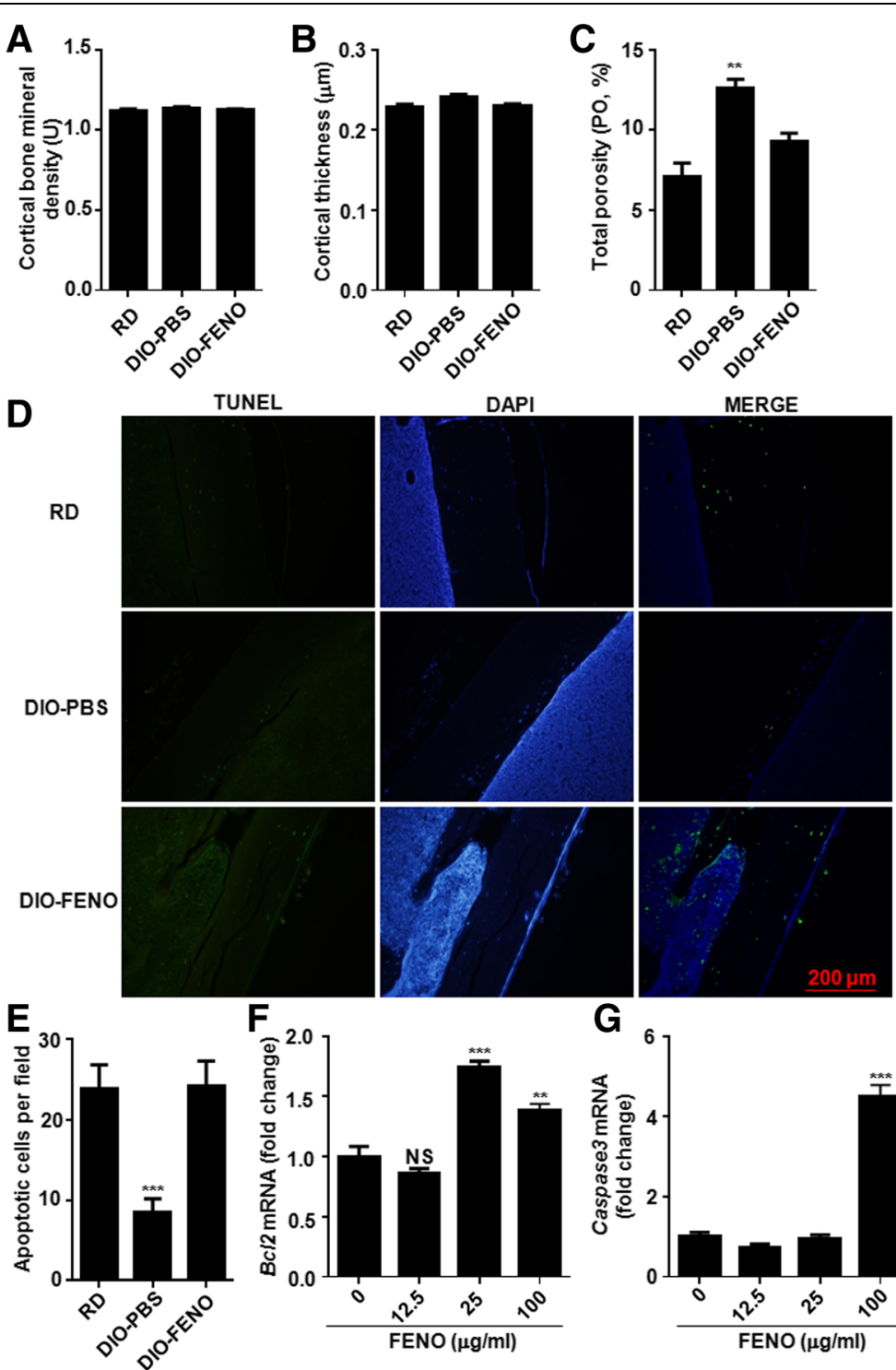

G
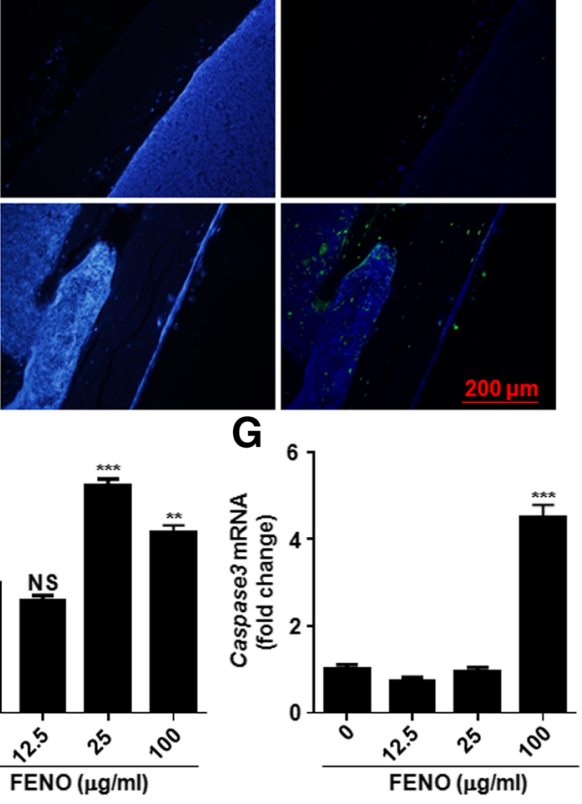

Fig. 2 HFD-induced T2DM mice treated with fenofibrate showed low cortical porosity. Analysis of a cortical bone mineral density, $\mathbf{b}$ cortical thickness, and c cortical porosity of femur $(n=7)$. $\mathbf{d}$ Representative sections of femur from different groups were stained with TUNEL, and $\mathbf{e}$ apoptotic cells per field were counted from 5 slides (10 fields in each slide). $\mathbf{f ~ B c l} 2$ and $\mathbf{g}$ caspase 3 expression in MC3T3 cells treated with fenofibrate at different concentrations detected by qPCR. Results are presented as mean \pm SD obtained from three repeated measurements. ${ }^{*} P<0.05,{ }^{* *} P<0.01$, ${ }^{* * *} P<0.001$ 
with $100 \mu \mathrm{g} / \mathrm{mL}$ fenofibrate (Fig. 2f and g). Therefore, we infer that fenofibrate might promote apoptosis of osteoblasts, resulting in a decrease in bone porosity.

\section{Fenofibrate causes a significant decrease in the expression of collagen I and osteocalcin in the HFD-induced T2DM mice}

In order to clarify the changes in extracellular matrix composition in the bones of HFD-induced T2DM mice, immunohistochemical staining was performed to evaluate the expression level of collagen I. The results showed that the expression of collagen I in DIO-PBS group was obvious lower than that in the RD group, while that in the DIO-FENO group was further reduced (Fig. 3a). Statistically, the expression of collagen I in the DIO-FENO group was significantly decreased than that in the RD group and the DIO-PBS group $(P<0.05)$ (Fig. 3b). Meanwhile, Western blot also found that the protein expression of collagen I and osteocalcin in the DIOFENO group was reduced (Fig. 3c, d). Therefore, fenofibrate may decrease the expression of collagen I and osteocalcin in osteoblasts extracellular matrix, thus affecting the bone structure and strength.

\section{High dose of fenofibrate may affect osteoblast activity by down-regulating Runx2}

To investigate how fenofibrate affects the expression of collagen I and osteocalcin in T2DM mice, in vitro experiments were performed using MC3T3 cells. CCK- 8 assay results showed that the cell viability was not significantly changed at the low concentration of fenofibrate $(0$ and
$25 \mu \mathrm{g} / \mathrm{ml}$ ) (Fig. 4a). At the low concentration of $12.5 \mu \mathrm{g} / \mathrm{ml}$, the osteocalcin and Spp1 genes were significantly increased, while at $25 \mu \mathrm{g} / \mathrm{mL}$, the Runx2 and Sp7 genes were increased greatly $(P<0.05$, Fig. $4 \mathrm{~b}-\mathrm{e})$. These results were similar to the finding that fenofibrate may regulate the differentiation of osteoblast through bone morphogenetic proteins [24]. However, this study was focused on the side effects of fenofibrate on the bones of T2DM mice with dyslipidemia. Thus, the protective mechanism of fenofibrate at low concentrations was not discussed deeply. Moreover, with the increase of concentration, the cell viability was decreased greatly at the high concentrations of fenofibrate (Fig. 4a). The expression of osteoblast markers was also significantly decreased. At the high concentration of $100 \mu \mathrm{g} / \mathrm{ml}$, the Runx2 mRNA levels were decreased greatly compared with the untreated cells $(P<0.05)$ (Fig. 4b). And Osteocalcin, Spp1, Spp7 were also decreased compared with the low concentrations of fenofibrate group (Fig. 4c-e). Therefore, it is concluded that high dose of fenofibrate may inhibit the expression of Runx 2 gene in osteoblasts, and thus have a negative effect on the bones of T2DM mice with dyslipidemia.

\section{Discussion}

T2DM is sometimes associated with skeletal system complications characterized by reduced bone mass and increased bone fragility $[1,2,23]$. In this study, we used HFD-induced diabetic mouse model to investigate the effects of fenofibrate, a lipid-lowering drug, on skeletal system during the treatment of diabetic dyslipidemia. It was found that fenofibrate could further reduce the
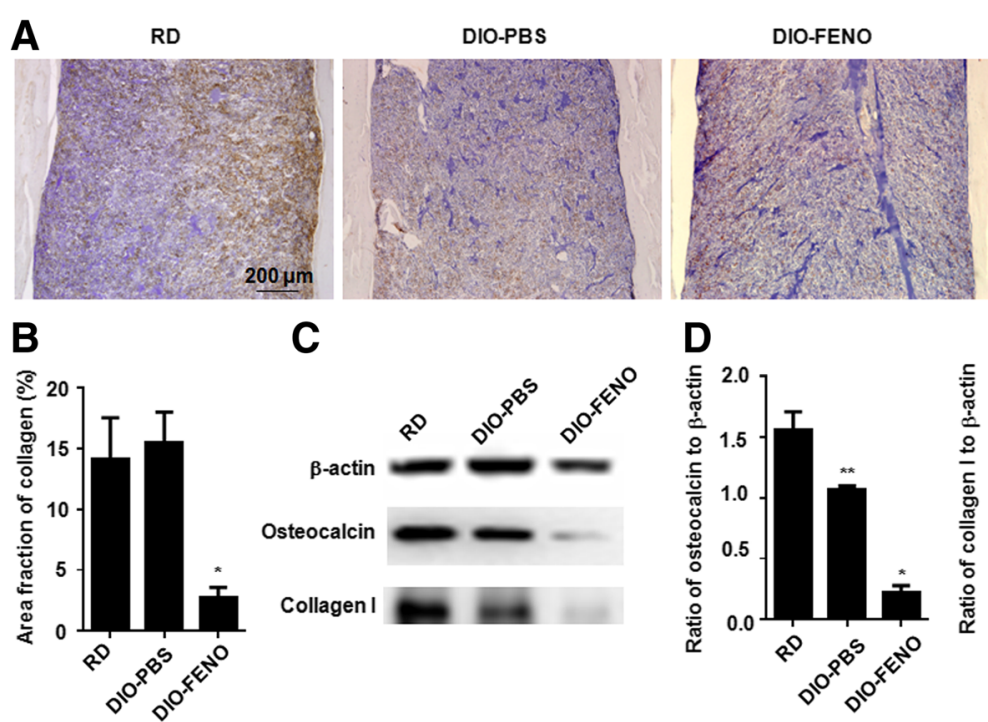

C
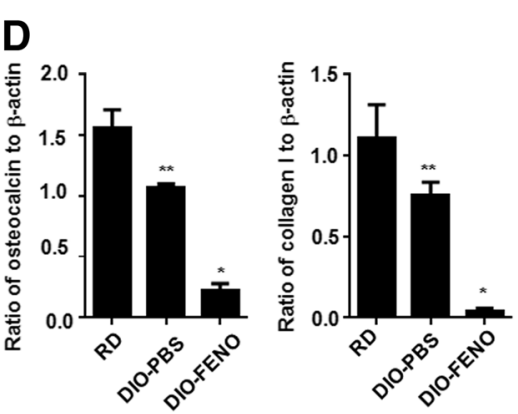

Fig. 3 HFD-induced T2DM mice treated with fenofibrate down regulated the expression of osteoblast extracellular matrix. a Immunohistochemical staining for Collagen I in representative sections of femur from different groups. Magnification was 100x. b Area fraction of collagen I was counted from 5 slides (10 fields in each slide). c, d Western blot analysis for Collagen I and osteocalcin protein expression extracted from the bones of different groups. $\beta$-actin was used as control. ${ }^{*} P<0.05,{ }^{* *} P<0.01,{ }^{* * *} P<0.001$ 


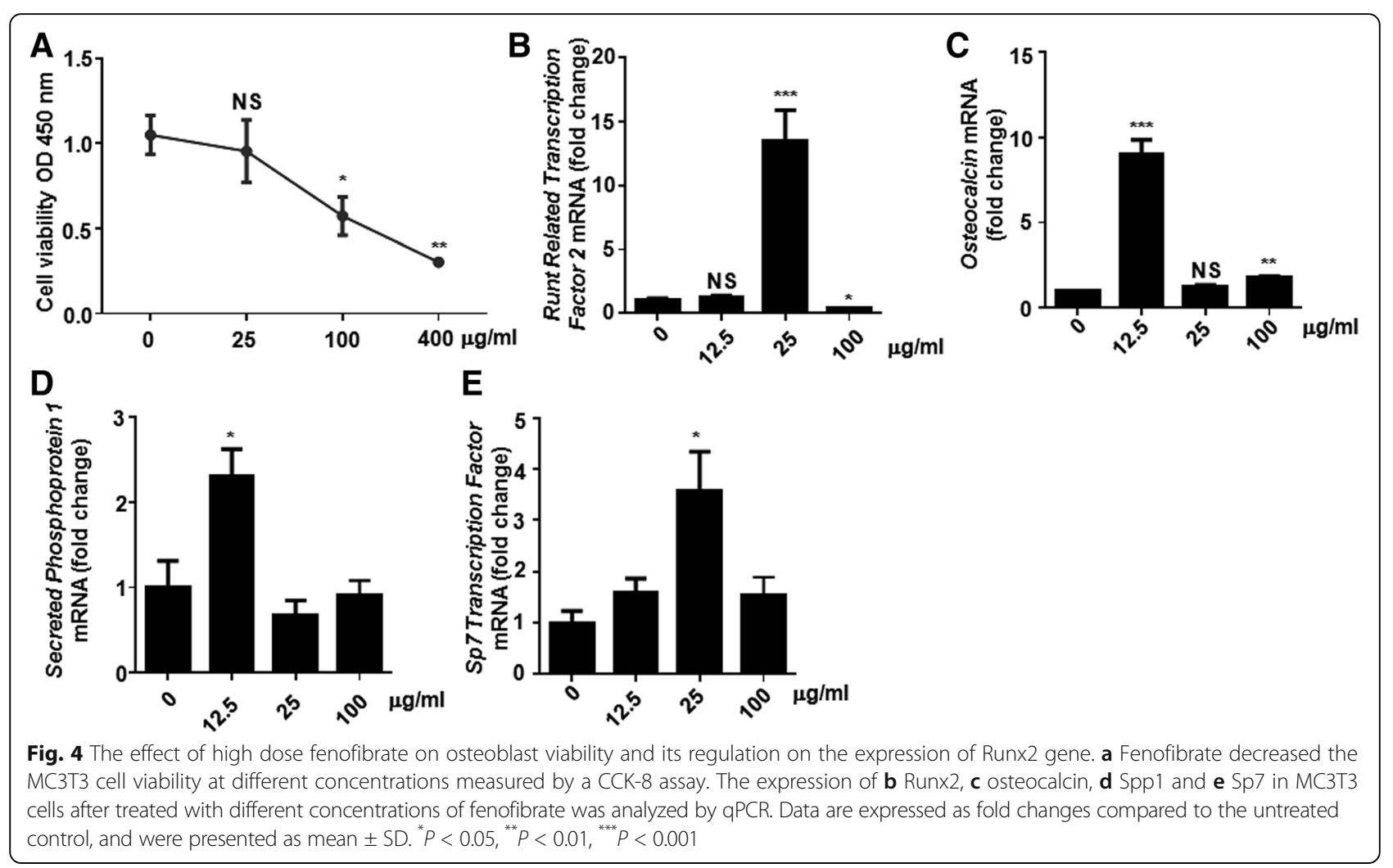

biomechanical parameters of T2DM mice by modulating the expression of Runx2, thereby increasing the risk of diabetic brittle fracture.

Brittle fracture is a common complication in T2DM patients, which is characterized by a normal or slightly increased BMD value and an increased risk of fracture [25]. In the HFD-induced T2DM mice, we found no significant difference in BMD between the DIO-PBS group and the RD group, while the biomechanical activity was significantly reduced, thus inducing skeletal fragile fracture. However, the mechanism of brittle fracture of T2DM is not entirely clear. The Health, aging, and body composition (HABC) research found that T2DM patients had increased serum levels of cystatin C, an index of impaired renal function, which might lead to vitamin D uptake disorder [26]. In this research, the osteocalcin expression in the bone tissues of DIO-PBS group and DIO-FENO group was significantly lower than that in the normal group. Diaz-Lopez et al. also reported a significant reduction in both uncarboxylated and carboxylated osteocalcin in the sera of patients with T2DM [27]. Decreased osteocalcin levels indicate that osteoblast activity in T2DM patients is inhibited [28].

At present, metformin, pioglitazone and other hypoglycemic agents are the clinically preferred drugs for treatment of T2DM [1, 13, 29, 30], however, a longterm and high-dose usage can also cause some side effects. DeFronzo et al. found that long-term use of metformin may lead to severe metformin-associated lactic acidosis in some patients, and may further damage their renal function [31]. Stunes et al. found that the use of pioglitazone may activate Peroxisome proliferator-activated receptor- $\gamma$ (PPAR $\gamma)$, aggravating the loss of bone mass in patients with T2DM [32]. In addition to increased blood glucose in patients with T2DM, dyslipidemia is also the cause of increased mortality in patients [13].

Fenofibrate is the commonly used drug for the treatment of dyslipidemia. FIELD study showed that fenofibrate can effectively reduce blood TG levels and reduce the risk of total cardiovascular events in T2DM patients [17]. Meanwhile, it can delay retinopathy [19], and inhibit hypertrophy of fat cells to alleviate insulin resistance [33, 34]. However, the usage of fenofibrate also causes some side effects like heartburn in primary biliary cirrhosis.

In this study, the number of trabecular bone was significantly reduced after fenofibrate intragastric treatment, and the max load was also decreased significantly. This indicates that the bone loss of T2DM mice is increased after taking fenofibrate. Fenofibrate is an important PPAR $\alpha$ agonist [34], and PPAR $\alpha$ is mainly involved in the body fat metabolism process, such as fatty acid uptake through cell membranes, fatty acid binding in cells, fatty acid oxidation and lipoprotein assembly and transport [35]. There are also related reports about the impact of PPAR $\alpha$ on bones. Stunes et al. found that 
fenofibrate can significantly alleviate the symptoms of osteoporosis, while pioglitazone increased bone loss and negatively regulated bone structure in the ovariectomized rat model [32]. The different result after lipidlowering drug treatment may depend on the duration of drug usage and the health of the bones [36]. However, in the HFD-induced T2DM model of this study, the expression of collagen I and osteocalcin in bone tissues was significantly decreased after fenofibrate treatment. High concentration of fenofibrate inhibited the expression of Runx2 and thus inhibited collagen I expression. These results are consistent with the study by Ortuño et al. that Runx2 can promote collagen I expression [37].

Burghardt et al. found that the cortical bone porosity of postmenopausal T2DM patients increased significantly [38], which may be one of the main reasons leading to the increase of bone brittleness. However, the mechanism of increased cortical bone porosity is still unclear. Lotinun et al. found that continuous subcutaneous injection of parathyroid hormone (PTH) can lead to increased cortical bone porosity [39], while Jilka et al. found increased femur trabecular bone mass together with significant increase in cortical bone porosity after knocking out the apoptosis-related genes, such as Bax and Bak, in mice [40]. This study confirmed that cell apoptosis in the cortical bones of T2DM mice was significantly lower than that of the RD group, and cell apoptosis increased significantly after fenofibrate treatment. This result is similar to the report by $\mathrm{Li}$ et al. that the apoptosis of triple-negative breast cancer cells increased after the addition of PPAR $\alpha$ agonist fenofibrate [41]. We also found that the expression of $\mathrm{Bcl} 2$ and caspase3 in osteoblast cell line MC3T3 significantly increased after treated with fenofibrate, compared to those in the untreated cells. This further confirmed that fenofibrate in different concentrations could promote osteoblast apoptosis and thus alleviate the increase in cortical bone porosity.

\section{Conclusions}

In conclusion, we successfully established T2DM mouse model using HFD. After treatment with fenofibrate, the number of trabecular bone was significantly lower, and the expression of collagen I and osteocalcin in bone tissues was significantly decreased. Although the cortical bone porosity had a certain decline compared with that in the model group, osteoblasts viability and biomarkers were significantly reduced after high-dose fenofibrate treatment. However, this cannot alleviate the reduction of bone strength. The reduced bone quality caused by fenofibrate might be due to the down-regulation of Runx2. This suggests that the skeletal system of diabetic patients should be monitored when using fenofibrate to treat dyslipidemia in the clinic, in order to prevent the development of brittle fractures.

\section{Abbreviations}

BMD: Bone mineral density; BV/TV: Bone volume per tissue volume; DIO: Dietinduced obese; FIELD: Fenofibrate Intervention and Event Lowering in Diabetes; HFD: High-fat-diet; LPL: Lipoprotein lipase; micro-CT: Microcomputed tomography; T2DM: Type 2 diabetes mellitus; TG: Triglyceride

\section{Acknowledgements}

Not applicable.

\section{Funding}

This work was supported by the Projects of International Cooperation and Exchanges NSFC (81420108021), NSFC (31371373), National Key Technology Support Program (2015BAI08B02), Excellent Young Scholars NSFC (81622033), the Nature Science Foundation of Jiangsu Province (BK20151395), the Natural Science Foundation of Jiangsu Province (Grants No BK20141088), the Open Fund of State Key Laboratory of Natural Medicines (No. SKLNMKF201606), Jiangsu Provincial Key Medical Center Foundation, Jiangsu Provincial Medical Talent Foundation, Jiangsu Provincial Medical Outstanding Talent Foundation and the Fundamental Research Funds for the Central Universities (021414380330).

\section{Availability of data and materials}

All data generated or analysed during this study are included in this published article.

\section{Authors' contributions}

$C L, B X$, and QJ conceived and designed the experiments. TS and KL performed the experiments. SS, QT, and KZ analyzed the data. XZ, YS, XL and HT contributed reagents/materials/analysis tools. $C L, B X, Q J$, TS and KL wrote the manuscript. All authors read and approved the final manuscript.

\section{Ethics approval}

All animal experiments were conducted in accordance with the Institutional Animal Ethics Committee and Animal Care Guidelines for the Care and Use of Laboratory Animals of Nanjing University.

Consent for publication

Not applicable.

\section{Competing interests}

The authors declare that they have no competing interests.

\section{Publisher's Note}

Springer Nature remains neutral with regard to jurisdictional claims in published maps and institutional affiliations.

\section{Author details}

${ }^{1}$ Department of Sports Medicine and Adult Reconstructive Surgery, Drum Tower Hospital, School of Medicine, Nanjing University, No. 321 Zhongshan Road, Nanjing 210008, People's Republic of China. ${ }^{2}$ State Key Laboratory of Pharmaceutical Biotechnology and Jiangsu Key Laboratory of Molecular Medicine and School of Medicine, Nanjing University, No. 22 Hankou Road, Gulou District, Nanjing, Jiangsu Province 210093, China. ${ }^{3}$ Joint Research Center for Bone and Joint Disease, Model Animal Research Center (MARC), Nanjing University, Nanjing 210093, China. ${ }^{4}$ State Key Laboratory of Natural Medicines, China Pharmaceutical University, Nanjing 210009, China. ${ }^{5}$ Liver Disease Collaborative Research Platform of Medical School of Nanjing University, Nanjing 210093, China.

Received: 26 August 2017 Accepted: 4 October 2017

Published online: 13 October 2017

\section{References}

1. DeFronzo RA, Ferrannini E, Groop L, Henry RR, Herman WH, Holst JJ, et al. Type 2 diabetes mellitus. Nat Rev Dis Primers. 2015;1:15019.

2. Chen L, Magliano DJ, Zimmet PZ. The worldwide epidemiology of type 2 diabetes mellitus-present and future perspectives. Nat Rev Endocrinol. 2011;8:228-36.

3. Soni SS, Gowrishankar S, Kishan AG, Raman A. Non diabetic renal disease in type 2 diabetes mellitus. Nephrology (Carlton). 2006;11:533-7. 
4. Schwartz AV, Vittinghoff E, Bauer DC, Hillier TA, Strotmeyer ES, Ensrud KE, et al. Association of BMD and FRAX score with risk of fracture in older adults with type 2 diabetes. JAMA. 2011;305:2184-92.

5. Bonds DE, Larson JC, Schwartz AV, Strotmeyer ES, Robbins J, Rodriguez BL, et al. Risk of fracture in women with type 2 diabetes: the Women's health initiative observational study. J Clin Endocrinol Metab. 2006;91:3404-10.

6. Bucala R, Vlassara H. Advanced glycosylation end products in diabetic renal and vascular disease. Am J Kidney Dis. 1995;26:875-88.

7. Hein GE. Glycation endproducts in osteoporosis-is there a pathophysiologic importance? Clin Chim Acta. 2006;371:32-6.

8. Saito M, Fujii K, Mori Y, Marumo K. Role of collagen enzymatic and glycation induced cross-links as a determinant of bone quality in spontaneously diabetic WBN/Kob rats. Osteoporos Int. 2006:17:1514-23.

9. Yamamoto M, Yamauchi M, Sugimoto T. Elevated sclerostin levels are associated with vertebral fractures in patients with type 2 diabetes mellitus. J Clin Endocrinol Metab. 2013;98:4030-7.

10. Weyer C, Funahashi T, Tanaka S, Hotta K, Matsuzawa Y, Pratley RE, et al. Hypoadiponectinemia in obesity and type 2 diabetes: close association with insulin resistance and hyperinsulinemia. J Clin Endocrinol Metab. 2001;86:1930-5.

11. Tamura T, Yoneda M, Yamane K, Nakanishi S, Nakashima R, Okubo M, et al. Serum leptin and adiponectin are positively associated with bone mineral density at the distal radius in patients with type 2 diabetes mellitus. Metabolism. 2007:56:623-8.

12. Johnston SS, Conner C, Aagren M, Ruiz K, Bouchard J. Association between hypoglycaemic events and fall-related fractures in Medicare-covered patients with type 2 diabetes. Diabetes Obes Metab. 2012;14:634-43.

13. Pillarisetti S. Potential drug combinations to reduce cardiovascular disease burden in diabetes. Trends Pharmacol Sci. 2016;37:207-19.

14. Rosenblit PD. Common medications used by patients with type 2 diabetes mellitus: what are their effects on the lipid profile? Cardiovasc Diabetol. 2016;15:95.

15. Bijelic R, Balaban J, Milicevic S. Correlation of the lipid profile, BMI and bone mineral density in postmenopausal women. Mater Soc. 2016;28:412-5.

16. Yamaguchi T, Sugimoto T, Yano S, Yamauchi M, Sowa H, Chen Q, et al. Plasma lipids and osteoporosis in postmenopausal women. Endocr J. 2002:49:211-7.

17. Keech A, Simes RJ, Barter P, Best J, Scott R, Taskinen MR, et al. Effects of long-term fenofibrate therapy on cardiovascular events in 9795 people with type 2 diabetes mellitus (the FIELD study): randomised controlled trial. Lancet. 2005:366:1849-61.

18. Rajamani K, Colman PG, Li LP, Best JD, Voysey M, D'Emden MC, et al. Effect of fenofibrate on amputation events in people with type 2 diabetes mellitus (FIELD study): a prespecified analysis of a randomised controlled trial. Lancet. 2009;373:1780-8.

19. Keech AC, Mitchell P, Summanen PA, O'Day J, Davis TM, Moffitt MS, et al. Effect of fenofibrate on the need for laser treatment for diabetic retinopathy (FIELD study): a randomised controlled trial. Lancet. 2007;370:1687-97.

20. Lee $\mathrm{YH}$, Lee $\mathrm{CH}$, Lee J. Effect of fenofibrate in combination with urate lowering agents in patients with gout. Korean J Intern Med. 2006;21:89-93.

21. Krishna SM, Seto SW, Moxon JV, Rush C, Walker PJ, Norman PE, et al. Fenofibrate increases high-density lipoprotein and sphingosine 1 phosphate concentrations limiting abdominal aortic aneurysm progression in a mouse model. Am J Pathol. 2012;181:706-18.

22. Mattila $P$, Knuuttila M, Kovanen V, Svanberg M. Improved bone biomechanical properties in rats after oral xylitol administration. Calcif Tissue Int. 1999;64:340-4.

23. Napoli N, Chandran M, Pierroz DD, Abrahamsen B, Schwartz AV, Ferrari SL, et al. Mechanisms of diabetes mellitus-induced bone fragility. Nat Rev Endocrinol. 2017:13:208-19.

24. Takano M, Otsuka F, Matsumoto Y, Inagaki K, Takeda M, Nakamura E, et al. Peroxisome proliferator-activated receptor activity is involved in the osteoblastic differentiation regulated by bone morphogenetic proteins and tumor necrosis factor-alpha. Mol Cell Endocrinol. 2012;348:224-32.

25. Kurra S, Fink DA, Siris ES. Osteoporosis-associated fracture and diabetes. Endocrinol Metab Clin N Am. 2014;43:233-43.

26. Schwartz AV, Vittinghoff E, Sellmeyer DE, Feingold KR, de Rekeneire $N$, Strotmeyer ES, et al. Diabetes-related complications, glycemic control, and falls in older adults. Diabetes Care. 2008:31:391-6.

27. Diaz-Lopez A, Bullo M, Juanola-Falgarona M, Martinez-Gonzalez MA, Estruch $\mathrm{R}$, Covas Ml, et al. Reduced serum concentrations of carboxylated and undercarboxylated osteocalcin are associated with risk of developing type 2 diabetes mellitus in a high cardiovascular risk population: a nested casecontrol study. J Clin Endocrinol Metab. 2013;98:4524-31.
28. Li H, Xie H, Liu W, Hu R, Huang B, Tan YF, et al. A novel microRNA targeting HDAC5 regulates osteoblast differentiation in mice and contributes to primary osteoporosis in humans. J Clin Invest. 2009;119:3666-77.

29. Turner LW, Nartey D, Stafford RS, Singh S, Alexander GC. Ambulatory treatment of type 2 diabetes in the U.S., 1997-2012. Diabetes Care. 2014;37:985-92.

30. Engebretson $\mathrm{S}$, Kocher T. Evidence that periodontal treatment improves diabetes outcomes: a systematic review and meta-analysis. J Clin Periodontol. 2013;40(Suppl 14):S153-63.

31. DeFronzo R, Fleming GA, Chen K, Bicsak TA. Metformin-associated lactic acidosis: current perspectives on causes and risk. Metabolism. 2016;65:20-9.

32. Stunes AK, Westbroek I, Gustafsson BI, Fossmark R, Waarsing JH, Eriksen EF, et al. The peroxisome proliferator-activated receptor (PPAR) alpha agonist fenofibrate maintains bone mass, while the PPAR gamma agonist pioglitazone exaggerates bone loss, in ovariectomized rats. BMC Endocr Disord. 2011:11:11.

33. Srivastava RA, He S. Anti-hyperlipidemic and insulin sensitizing activities of fenofibrate reduces aortic lipid deposition in hyperlipidemic Golden Syrian hamster. Mol Cell Biochem. 2010;345:197-206.

34. Jeong S, Yoon M. Fenofibrate inhibits adipocyte hypertrophy and insulin resistance by activating adipose PPARalpha in high fat diet-induced obese mice. Exp Mol Med. 2009;41:397-405

35. Everett L, Galli A, Crabb D. The role of hepatic peroxisome proliferatoractivated receptors (PPARs) in health and disease. Liver. 2000;20:191-9.

36. Meier C, Schwartz AV, Egger A, Lecka-Czernik B. Effects of diabetes drugs on the skeleton. Bone. 2016;82:93-100.

37. Ortuno MJ, Susperregui AR, Artigas N, Rosa JL, Ventura F. Osterix induces Col1a1 gene expression through binding to Sp1 sites in the bone enhancer and proximal promoter regions. Bone. 2013;52:548-56.

38. Burghardt AJ, Issever AS, Schwartz AV, Davis KA, Masharani U, Majumdar S, et al. High-resolution peripheral quantitative computed tomographic imaging of cortical and trabecular bone microarchitecture in patients with type 2 diabetes mellitus. J Clin Endocrinol Metab. 2010;95:5045-55.

39. Lotinun S, Evans GL, Bronk JT, Bolander ME, Wronski TJ, Ritman EL, et al. Continuous parathyroid hormone induces cortical porosity in the rat: effects on bone turnover and mechanical properties. J Bone Miner Res. 2004;19:1165-71.

40. Jilka RL, O'Brien CA, Roberson PK, Bonewald LF, Weinstein RS, Manolagas SC. Dysapoptosis of osteoblasts and osteocytes increases cancellous bone formation but exaggerates cortical porosity with age. J Bone Miner Res. 2014;29:103-17.

41. Li T, Zhang Q, Zhang J, Yang G, Shao Z, Luo J, et al. Fenofibrate induces apoptosis of triple-negative breast cancer cells via activation of NF-kappaB pathway. BMC Cancer. 2014;14:96.

\section{Submit your next manuscript to BioMed Central and we will help you at every step:}

- We accept pre-submission inquiries

- Our selector tool helps you to find the most relevant journal

- We provide round the clock customer support

- Convenient online submission

- Thorough peer review

- Inclusion in PubMed and all major indexing services

- Maximum visibility for your research

Submit your manuscript at www.biomedcentral.com/submit
) Biomed Central 